inappropriate if the sequence differed from the pictogram. Ethics approval was obtained before starting the chart review.

Results. A total of 120 patients were identified pre-/postintervention. 20\% (10/51) had received $\mathrm{AB}$ in an incorrect sequence prior to the pictogram implementation compared with $11 \%(8 / 70)$ postintervention. AB prescribed were piperacillin/tazobactam (24\%), azithromycin (24\%) and vancomycin (18\%), ceftriaxone (15\%) for sepsis arising from pneumonia, urinary tract, and intra-abdominal infections.

Conclusion. The availability of a pictogram to guide the sequence of antibiotic administration in the septic patient can assure its correct administration sequence and potentially affect patient outcomes. An improvement $(45 \%, P=0.2)$ was seen post implementation suggesting the pictogram to be a helpful visual aid for nurses. Although not statistically significant, the difference implies a tendency that may be explored in a larger sample size to search for a potential effect.

References

1. Rhodes A, et al. Surviving Sepsis Campaign: International Guidelines for Management of Sepsis and Septic Shock 2016. Crit Care Med 2017(45)486-552.

2. Roberts $\mathrm{R}$, et al. Impact of Antibiotic Initiation Sequence on Outcomes in Patients with Septic Shock. Poster 652. Crit Care Med 2016(44) Suppl.

\section{Prioritization of Antibiotic} Administration for STAT orders for the septic patient

- Verify patient's allergy status to antibiotics and advise prescribing physician if an allergy is

- Obtain blood cultures as quickly as possible, before administration of antibiotics

- Administer 1 antibiotic at a time. If an allergic reaction were to occur, the offending agent may be easier to identify.

1. I $f$ both an antibacterial and an antifungal are prescribed, initiate the antibacterial first unless otherwise indicated by the prescriber

Exception: If Clindamycin and Penicillin $G$ re prescribedfor a complicated skin and soft tissue infection (CSSTI), administer agent simultaneously in Y-site.
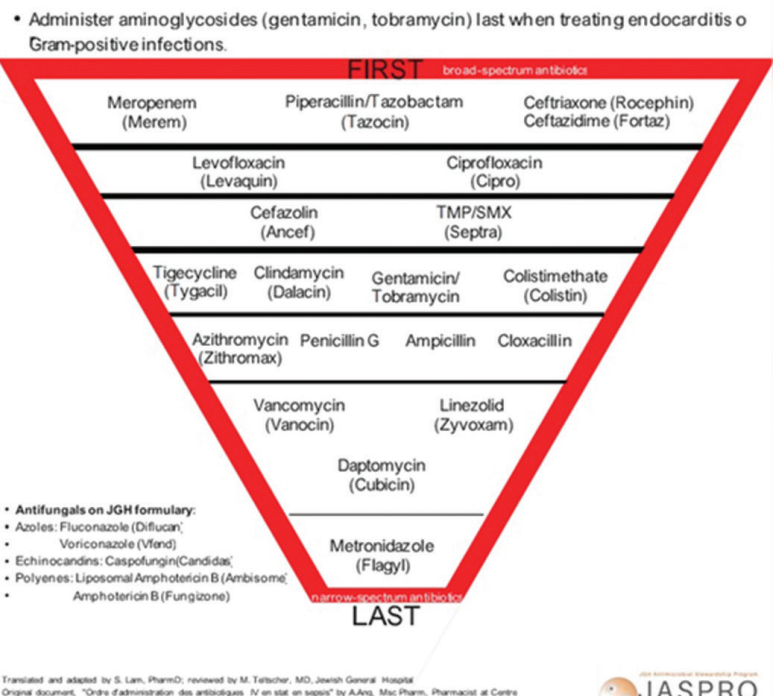

JASPRO

Disclosures. All authors: No reported disclosures.

244. Impact of Antibiotic Susceptibility Reporting on Broad-Spectrum Antibiotic Use in Serratia and Morganella Bacteremia

Wendy Hui Wen Ng, BSc (Pharm) (Hons) ${ }^{1}$; Joy Yong, BSc (Pharm) (Hons), BCPS ${ }^{1}$; Ka Lip Chew, MBBS, B. Med. Sci. FRCPath ${ }^{2}$ and Janice Li, MClin Pharm, BCPS ${ }^{1}$ Pharmacy, National University Hospital, Singapore, Singapore, ${ }^{2}$ Department of Laboratory Medicine, National University Hospital, Singapore, Singapore

Session: 51. Antimicrobial Stewardship: Interventions to Improve Outcomes Thursday, October 4, 2018: 12:30 PM

Background. The increase in antibiotic resistance highlights the necessity of antimicrobial stewardship strategies to guide antibiotic de-escalation. Retrospective data suggest noninferior outcomes with carbapenem-sparing regimens in AmpC- $\beta$-lactamase producing Serratia marcescens and Morganella morganii bacteremia. To minimize broad-spectrum (BS) antibiotic use, our microbiology laboratory recently changed the antibiotic susceptibility reporting for these organisms in blood cultures to include narrow spectrum third-generation cephalosporins. We sought to assess the impact of this change on BS antibiotic use and clinical outcomes. We also sought to compare outcomes between BS and narrow spectrum antibiotics in bacteremia caused by these organisms.

Methods. We retrospectively reviewed all adult patients with Serratia marcescens or Morganella morganii in blood culture 2 years pre- and post-change of susceptibility reporting from February 13-February 17. Exclusion criteria included: (1) $>1$ pathogen found in blood culture, (2) no antibiotic treatment given, (3) death within 48 hours of positive blood culture. The rates of broad-spectrum antibiotic use between the two periods were compared. Secondary outcomes evaluated included in-hospital mortality, clinical response, and microbiologic success.

Results. A total of 112 patients were screened. There were 30 patients prechange and 46 patients postchange of reporting included after exclusions. Patients in both groups had similar baseline characteristics. There was a decrease in BS antibiotic use postchange $(70 \%$ to $54.3 \%)$ although this was not statistically significant $(P=0.172)$. Specifically, cefepime use had decreased significantly from $46.7 \%$ to $6.5 \%(P<0.001)$ No significant differences were observed in secondary outcomes between patients pre- and postchange. The use of BS antibiotics was significantly associated with higher in-hospital mortality $(100 \%$ vs. $55.2 \%, P=0.010)$. No mortality was observed in patients on narrow spectrum antibiotics.

Conclusion. BS antibiotic use, specifically cefepime, was reduced after susceptibility reporting changes without affecting outcomes in Serratia marcescens and Morganella morganii bacteremia. This demonstrates the potential role of selective susceptibility reporting in antimicrobial stewardship.

Disclosures. All authors: No reported disclosures.

245. Elimination of Routine Urinalysis Before Elective Orthopedic Surgery Reduces Antibiotic Utilization Without Impacting Catheter-Associated Urinary Tract Infection or Surgical Site Infection Rates

Megan Hoffman, (undergraduate) ${ }^{1,2}$; Kevin Counterman, $\mathrm{BS}^{2}$; Sheila Querfurth, MSN, ANP-BC ${ }^{1}$; Brian L. Hollenbeck, MD ${ }^{3,4}$; Christine Bell, MSN, RN, CAPA, WCC and New England Baptist Hospital Antimicrobial Stewardship Committee; ${ }^{1}$ New England Baptist Hospital, Boston, Massachusetts, ${ }^{2}$ Northeastern University, Boston, Massachusetts, ${ }^{3}$ Division of Infectious Diseases, New Engalnd Baptist Hospital, Boston, Massachusetts, ${ }^{4}$ Medicine, Harvard Medical School, Boston, Massachusetts

Session: 52. Antimicrobial Stewardship: Special Populations

Thursday, October 4, 2018: 12:30 PM

Background. Routine preoperative urinalysis testing has been common practice for the surgical orthopedic population for decades regardless of the presence of symptoms. Studies demonstrate low concordance between bacteria cultured from the surgical wound and those cultured in the urine. In addition, antibiotic overuse is costly and promotes antibiotic resistance. We developed a quality improvement initiative aimed at reducing unnecessary antibiotic use by (1) modifying testing algorithms to target symptomatic and high-risk patients (2) modifying reflux to culture criteria, and (3) developing treatment guidelines for providers.

Methods. This pre/poststudy utilized data from our hospital's orthopedic registry to identify all hip and knee arthroplasty and spine fusion procedures for a prepractice and postpractice change period. Univariate analysis compared groups for differences in age, gender diabetes, procedure mix, foley catheter utilization, and other variables that may affect the outcomes. Surgical Site Infection (SSI) and Catheter-Associated Urinary Tract Infection (CAUTI) rates were extracted from infection control databases. Urinalysis results, urine culture results, and antibiotic prescriptions were collected retrospectively from every 10th chart from the pre-period, and measured prospectively on all patients for the post-period.

Results. A total of 9,949 people met criteria for inclusion in the study. Baseline demographics were similar between study groups, with the exception of catheter-utilization which decreased by 0.06 device days/patient day between periods $(P<0.05)$. During the pre-practice change period, $160 / 5,340(3.0 \%)$ patients were treated preoperatively for a positive urine culture, 40/160 (25\%) of whom had urinary symptoms. During the post-practice change period, $10 / 4,609(0.2 \%)$ patients were treated preoperatively $(P<$ $0.05)$, all of whom had urinary symptoms. The SSI rate was $0.34 / 100$ procedures in the pre-period and $0.17 / 100$ procedures in the post period $(P=0.11)$. One patient in the pre-period and one patient in the post-period developed CAUTI $(P=0.92)$.

Conclusion. Elimination of routine urinalysis before orthopedic surgery resulted in no change in SSI or CAUTI rates, but $93 \%$ reduction in antibiotic utilization.

Figure 1: Impact of prescreening urinalysis practice change in an elective orthopedic population UA: urinalysis, UC: urine culture

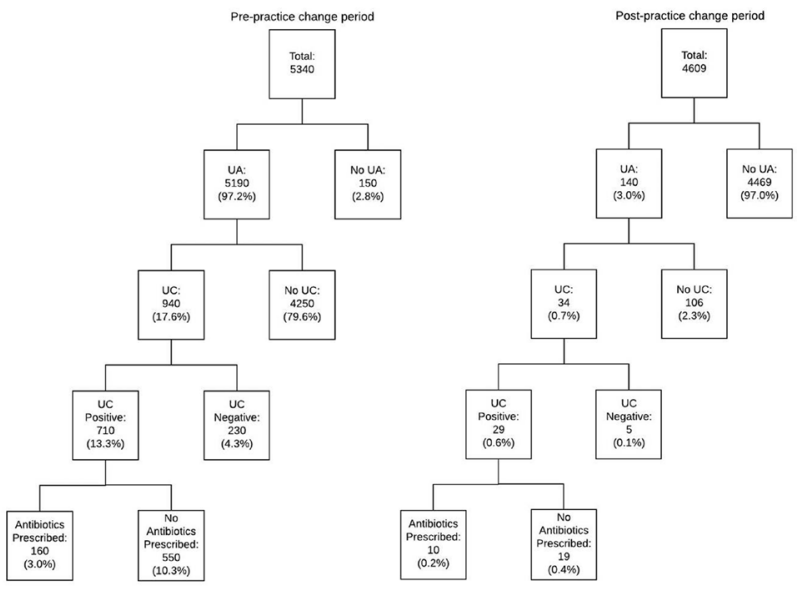

